\title{
In Situ Seedling Baiting to Isolate Plant Growth- Promoting Fungi From Dendrobium Officinale, An Over-Collected Medicinal Orchid In China
}

\author{
De-Yun Chen \\ Yunnan University \\ Xin-Ju Wang \\ Yunnan University \\ Tai-Qiang Li \\ Yunnan University \\ Neng-Qi Li \\ Yunnan University \\ Jiang-Yun Gao ( $\nabla$ jiangyun.gao@ynu.edu.cn ) \\ Yunnan University https://orcid.org/0000-0003-1541-5922
}

Research article

Keywords: In situ seedling baiting, Mycorrhizal fungi, Orchid conservation, Seedling growth, Traditional Chinese medicine, Tulasnella

Posted Date: January 15th, 2021

DOl: https://doi.org/10.21203/rs.3.rs-144221/v1

License: (c) (1) This work is licensed under a Creative Commons Attribution 4.0 International License. Read Full License 


\section{Abstract}

Background: Orchids are highly dependent on mycorrhizae for survival and growth. Traditionally, to obtain orchid mycorrhizal fungi (OMFs) for plant growth, fungi have been isolated from the roots of mature wild plants; however, the species of OMF may change as the plants undergo different developmental stages. In this study, we developed the idea of using in situ seedling baiting to capture seedling growth-promoting fungi from Dendrobium officinale, an overcollected medicinal orchid in China.

Results: In vitro-produced seedlings of $D$. officinale were transplanted into their original habitats, and newly established roots of well-growing seedlings were sampled for fungal isolation. Roots were sampled at 6 different times over one year, and five Tulasnella species and one Fusarium species were obtained and identified morphologically and molecularly. The ability to promote seedling growth was tested in three Tulasnella species TPYD-1, TPYD-2 and TPYD-3, with high isolation frequencies by inoculation onto in vitro-produced $D$. officinale seedlings. Although the three fungi were closely related species and clustered together in the phylogenetic tree, they showed different efficiencies in promoting $D$. officinale seedling growth. Tulasnella TPYD-2 showed a strong ability to promote seedling growth and could be selected for use in restoration plantings of $D$. officinale.

Conclusions: Our results suggest that using an in situ seedling baiting technique could be an efficient way to obtain seedling growth-promoting fungi, and this approach could have broad applications in orchid mycorrhiza studies and orchid conservation.

\section{Background}

The genus Dendrobium Swartz is one of the largest genera in Orchidaceae, comprising approximately 1,100 species distributed in the tropical and subtropical areas of Asia and Australia, and approximately 78 species, including 14 endemic species, are native to China [1]. Of these, more than 40 Dendrobium species have a very long history of use in traditional Chinese medicine (TCM) and are known as Shi-Hu in Chinese [2]. Consequently, most of these medicinal Dendrobium species have been exploited to the point of local extirpation, especially in some "hotspot" areas $[3,4]$.

Increasing supplies is considered an important method to reduce harvesting pressures of overexploited wild species [5]. For medicinal Dendrobium species, the Shi-Hu industry has developed rapidly in southern China since the 1990s as a result of massive commercial cultivation; however, due to doubts about the quality and efficacy of the products obtained with this cultivation method, the lack of product standards and the low quantities of products, the utilization of these products in TCM is limited [6]. Moreover, such massive commercial cultivation has not alleviated pressure on wild populations, according to recent surveys on the orchid trade in China [7]. This reflects a consumer preference for wild-collected materials and the fact that wild collection maintains the livelihoods of local suppliers who do not have the capital to invest in rearing facilities. Preference for wild-collected medicines by Chinese consumers reflects the belief that these materials are more effective and contaminant-free than cultivated materials [8]. To 
balance the conservation of threatened Dendrobium species and the continuing demand for wild-grown materials, a restoration-friendly cultivation model for medicinal orchids, especially Dendrobium species, has been proposed, in which orchids are planted in natural settings[3, 8]; this method is considered promising for linking the commercial TCM industry with biodiversity conservation initiatives in China [6].

In the context of restoration-friendly cultivation, future scientific research on Dendrobium species, including mycorrhizal technology and microbial fertilizers, is urgently needed [6]. In our previous studies focused on the conservation of over-collected Dendrobium species, we obtained germination-enhancing fungi for different Dendrobium species through an in situ/ ex situ seed baiting technique [9-12], and the fungi have also been successfully used in restoration plantings of medicinal Dendrobium species [13]. However, the dynamics of fungal associations at the seedling stage remain largely unknown. In practice, we found that after seedling establishment, seedling growth varied greatly even in a small-scale environment [13], indicating that new fungal partners are established with seedlings and play an important role in plant growth. Orchids have complex symbioses with fungi throughout their lifespan, and the species of orchid mycorrhizal fungi (OMFs) may change in different plant developmental stages [14, 15]. Under natural conditions, the broadening and/or changing of a mycorrhizal association may enable orchids to adapt to the varied physiological changes during seedling development, including the switch to partial or full autotrophy, an increase in transpiration, or environmental fluctuations [16].

Motivated by the success of in situ/ex situ seed baiting, in this study, we developed the idea of using in situ seedling baiting to obtain seedling growth-promoting fungi, in which in vitro-produced seedlings of $D$. officinale were transplanted into their original habitats, and newly established roots of well-growing plants were sampled for fungal isolation. The aim of this study was to understand fungal diversity at the seedling stage and obtain OMFs for seedling growth to support restoration plantings of $D$. officinale. Here, we present our results, addressing three principal questions: (1) Does this method work to obtain OMFs associated with seedlings of $D$. officinale? (2) What are the effects of the dominant OMFs on seedling growth in D. officinale? (3) Have the OMFs obtained in the current study also been obtained in other studies in which OMFs were isolated from roots of mature wild plants, and do these fungi have positive effects on seedling growth in $D$. officinale?

\section{Results}

\section{Fungal isolation and identification}

In total, 64 purified fungal strains were successfully obtained from 600 root fragments of $D$. officinale that were sampled at 6 different times. All fungal strains were morphologically and molecularly identified as six fungal species, including five Tulasnella species and one Fusarium species (Additional file 1; Table 1). Among the 5 Tulasnella species, TPYD-1, TPYD-2, and TPYD-3 were isolated 15, 12 and 7 times and were present in 6, 5 and 3 samples, respectively (Fig. 1D-F), while TPYD-4 and TPYD-5 were isolated three and one times, respectively, and were only present in one sample each. Fusarium oxysporum TPYD6 was isolated from all 6 samples with a high isolation frequency of 26 out of 64 times (Additional file 1). 
Table 1

Molecular identification of six fungal species isolated from roots of Dendrobium officinale seedlings.

\begin{tabular}{|lllllll|}
\hline Fungus & $\begin{array}{l}\text { Total } \\
\text { isolation } \\
\text { times }\end{array}$ & $\begin{array}{l}\text { GenBank } \\
\text { accession } \\
\text { number }\end{array}$ & Close relative & $\begin{array}{l}\text { Accession } \\
\text { number }\end{array}$ & Ident & References \\
\hline $\begin{array}{l}\text { TPYD- } \\
1\end{array}$ & 15 & MN545675 & Tulasnella sp. & EF127682.1 & $98.82 \%$ & - \\
\hline $\begin{array}{l}\text { TPYD- } \\
2\end{array}$ & 12 & MN545849 & Tulasnella sp. & KP050605.1 & $97.42 \%$ & - \\
\hline $\begin{array}{l}\text { TPYD- } \\
3\end{array}$ & 7 & MN545858 & $\begin{array}{l}\text { Tulasnella } \\
\text { deliquescens }\end{array}$ & LC175331.1 & $97.67 \%$ & [62] \\
\hline $\begin{array}{l}\text { TPYD- } \\
4\end{array}$ & 3 & MN545859 & $\begin{array}{l}\text { uncultured } \\
\text { Tulasnellaceae }\end{array}$ & GQ241817.1 & $99.83 \%$ & [63] \\
\hline $\begin{array}{l}\text { TPYD- } \\
5\end{array}$ & 1 & MN545860 & $\begin{array}{l}\text { Tulasnella sp. } \\
\text { DerV }\end{array}$ & MK555332.1 & $99.33 \%$ & [11] \\
\hline $\begin{array}{l}\text { TPYD- } \\
6\end{array}$ & 26 & MN545304 & $\begin{array}{l}\text { Fusarium } \\
\text { oxysporum MC- }\end{array}$ & KU527806.1 & $99.23 \%$ & [64] \\
\hline
\end{tabular}

\section{Phylogenetic analysis}

A total of 28 fungal species/strains from 11 studies were reported to have positive effects on seedling growth in $D$. officinale (Additional file 2). Among them, 12 fungi were isolated from the protocorms or roots of $D$. officinale, 10 fungi were isolated from the roots of other Dendrobium species, and 6 fungi were obtained from the roots of non-Dendrobium orchid species. Interestingly, 17 fungi were OMFs, and the other 11 fungi were non-OMFs (Additional file 2). The ITS-rDNA sequences of 21 out of 28 fungi were downloaded from the GenBank database and used to generate the phylogenetic tree with the fungal sequences of five OMFs obtained in the current study. In the phylogenetic tree, the fungi TPYD-1, TPYD-2 and TPYD-3 were clustered together, while TPYD-4 and TPYD-5 were scattered with other species of Tulasnella and Epulorhiza (Fig. 2).

\section{Testing the capacity of fungi to support seedling growth}

In the incubation experiments, the three fungi TPYD-1, TPYD-2 and TPYD-3 with high isolation frequencies were examined in terms of their capacity to support seedling growth in $D$. officinale. At 30 and $60 \mathrm{~d}$ after incubation, no pelotons were observed in the roots under all treatments (Fig. 1G), and only scarce pelotons could be found in the roots under the TPYD-1 and TPYD- 2 treatments at $90 \mathrm{~d}$. All three fungi clearly started to colonize the roots from $120 \mathrm{~d}$ after incubation, and the numbers of pelotons in the roots under the three fungal treatments increased greatly at 150 and $180 \mathrm{~d}$ (Fig. 1H). 
All three fungi showed positive effects on seedling growth (Fig. 3A-E). The lengths of the longest roots in all three fungal treatment groups were significantly longer than that in the control group $(F=4.970, P=$ 0.003 ) with the highest growth rate in the TPYD-2 treatment ( $R=29.78 \%$; Fig. 3A). TPYD-1 and TPYD-2 significantly increased the biomass of seedlings as the fresh and dry weights in the two treatments were significantly higher than those in the control treatment (Fig. 3D \& E). However, there were no significant differences in the longest leaf length $(F=1.676, P=0.174$; Fig. $3 B$ ) or plant height $(F=3.212, P=0.205$; Fig. 3C) among all treatments.

150 days after incubation: At this stage, all three fungi significantly increased the root length and dry weight compared with the control treatment (Fig. 3F \& J), and the lengths of the longest leaf in the TPYD2 and TPYD-3 treatment groups were significantly longer than in the control group (Fig. 3G). Moreover, TPYD-2 showed very positive effects on seedling growth among all treatments, as all five measured indices were significantly higher in the TPYD-2 treatment than in the control treatment, and the plant height (growth rate: $H=36.44 \%$ ) and fresh weight (growth rate: $F=73.27 \%$ ) in the TPYD-2 treatment were significantly higher than in other two fungal treatments (all $P<0.001 ;$ Fig. $3 \mathrm{H} \& \mathrm{l}$ ).

180 days after incubation: Clearly, TPYD-2 promoted seedling growth more effectively than TPYD-1 and TPYD-3 (Fig. 11; Fig. 3K \& 0). Except for the longest leaf length, the longest root length, plant height, fresh weight and dry weight were significantly longer or higher in the TPYD-2 treatment than in the TPYD-1 and TPYD-3 treatments and the control treatment (all $P<0.001$ ). For the longest leaf length, there were no significant differences between the TPYD-2 and TPYD-3 treatments $(P=0.204)$, but the leaves were significantly longer than those in the TPYD-1 and control groups (all $P<0.05$; Fig. $3 \mathrm{~L}$ ). The biomasses of seedlings were also significantly higher under the TPYD-3 treatment than under the control (fresh weight: $P<0.001$; dry weight: $P=0.018$; Fig. 3 N \& 0 ).

\section{Discussion}

Orchid mycorrhizae are symbiotic associations between orchid plant roots and fungi, in which fungal hyphae grow within living plant cells and form intracellular pelotons $[17,18]$. Orchid mycorrhizal fungi (OMFs) are considered to belong to the so-called rhizoctonia aggregate, a polyphyletic group of fungi belonging to Tulasnellaceae, Ceratobasidiaceae, and Serendipitaceae [19]. Orchids are highly dependent on mycorrhizae for survival and growth: OMFs not only supply carbohydrates to facilitate the growth of nonphotosynthetic protocorms during the seed germination stage but also provide nutrients and growth factors to plants, conferring plant metal tolerance and inhibiting the development of pathogens $[17,20-$ 23].

Mycorrhizal technologies and microbial fertilizers could simplify orchid seedling production and promote plant growth and have been considered important aids to the sustainable development of the Dendrobium industry in China $[6,24,25]$. As the most popular medicinal plant species in the genus Dendrobium, D. officinale has received much research attention regarding its mycorrhizal symbionts [25]. Traditionally, to obtain the optimal source of fungal mycobionts for symbiotic germination or plant 
growth, fungi have been isolated and screened from the roots of mature wild plants [26, 27]. Among the 28 fungal species reported in $11 \mathrm{D}$. officinale studies, only five fungal species in one study were obtained from protocorms via ex situ seed baiting [28], while the other 23 fungi in the remaining 10 studies were isolated from the roots of wild mature plants, including $D$. officinale and other orchid species (Additional file 2). The 28 fungal species/strains, originally obtained from different orchid species and belonging to a wide range of taxonomic groups (17 OMFs and 11 non-OMFs), were found to have positive effects on the growth of $D$. officinale seedlings (Additional file 2). Seemingly, a randomly obtained fungus could promote seedling growth in $D$. officinale, however, all of the above fungi were tested in vitro, and there have been no reports on the successful application of these fungi in practice so far. Theoretically, a high diversity of fungi may associate with the roots of an adult orchid plant, an orchid will utilize different OMFs at different life history stages, and the mycorrhizal symbionts may change in different developmental stages $[14,15,29,30]$. It is unclear whether the fungi involved in the seedling stage remain until the plants reach adulthood in $D$. officinale.

The in situ/ex situ seed baiting technique has been suggested as an effective and easy way to obtain seed germination-enhancing fungi [31, 32]. Using this method, we successfully obtained efficient germination-enhancing fungi for different Dendrobium species [9-12], as well as other terrestrial orchids $[33,34]$. This led to the development of the idea of using seedling-trap experiments to capture seedling growth-promoting fungi in the current study. After in vitro-produced seedlings of $D$. officinale were transplanted for more than one year in their original habitats, newly established roots of well-growing plants were sampled six times across one year in different seasons (Additional file 1). In total, five OMFs and one non-OMFs were obtained and identified (Table 1). Tulasnella species TPYD-1 and TPYD-2 were the dominant OMFs with a higher frequency of isolation than other OMFs, and TPYD-1 was present in all six samples, while TPYD-2 was present in five samples (Additional file 1). The three fungi TPYD-1, TPYD2 and TPYD-3 with high isolation frequencies were closely related species, as they were clustered together in the phylogenetic tree (Fig. 2). Fusarium oxysporum TPYD-6 was also dominant, with a high isolation frequency and presence among the samples (Additional file 1).

In this study, to test and screen the fungi that could be used in real practice for growth of $D$. officinale seedlings, in vitro-produced seedlings were moved to mixed cultivation substrates in open environments, in which seedlings normally undergo an acclimatization stage. This could be a possible reason why the three fungi TPYD-1, TPYD-2 and TPYD-3 started to colonize seedling roots after a long period of $120 \mathrm{~d}$. However, the corresponding increases in pelotons in the roots and in the five measured indices in all three fungal treatments at 150 and $180 \mathrm{~d}$ suggested that all three fungi could promote seedling growth in $D$. officinale but showed different efficiencies (Fig. 3). At $180 \mathrm{~d}$ after incubation, the longest root length, plant height, fresh weight and dry weight of seedlings in the TPYD-2 treatment were significantly longer or higher than those in the other two fungal treatments and the control treatment (Fig. 3). TPYD-2 could be selected as an ideal OMF for seedling growth in the restoration-friendly cultivation of $D$. officinale.

Tulasnellaceae is regarded as one of the main fungal families of orchid mycorrhizae, and many of these species are found to support seed germination and seedling growth in Dendrobium species $[9,10,19,24]$. 
The other two Tulasnella species, TPYD-1 and TPYD-3, were closely related to TPYD-2 but showed less pronounced effects on seedling growth in $D$. officinale. Seedlings of $D$. officinale might need a longer time to acclimatize to fungal infection [35], or the two fungi might have other ecological functions [21, 22]. In the current study, we focused on the effects of fungi obtained from seedling baiting on seedling growth, but it would also be worthwhile to explore whether these fungi could effectively promote seed germination in $D$. officinale. In another study, we conducted comparisons of symbiotic germination of seeds inoculated with the TPYD-2 obtained in this study and six other fungal strains isolated from protocorms of $D$. officinale via in situ seed baiting. At $90 \mathrm{~d}$ after incubation, the percentage of seedlings in the LQ treatment was $70.09 \pm 3.2 \%$, while no protocorms or seedlings were found in the TPYD-2 treatment (Wang et al. unpublished data). The results also suggested that $D$. officinale could associate with different fungi in different life stages.

In addition to OMFs, other root-associated nonmycorrhizal endophytes have also been recorded and identified from a wide range of orchid species [36, 37]. In this study, Fusarium oxysporum TPYD-6 was isolated from all 6 samples with high isolation rates. Fusarium species have been reported to be associated with different orchid species [33, 38]. Interestingly, although Fusarium species have been reported as pathogens in many orchid species [39], especially F. oxysporum, which was reported to cause wilt disease in D. officinale [40], other studies have suggested that Fusarium species can stimulate seed germination in the terrestrial orchid Cypripedium reginae [41] and enhance resistance to pathogens and promote plant growth in Dendrobium species $[42,43]$. In a recent study, F. oxysporum KB-3, obtained from the roots of Bletilla striata, was considered an OMF because it could promote seed germination of $B$. striata, establish colonization and produce coiled hyphal structures within the cortical cells in the roots of B. striata and Dendrobium candidum [44]. As noted in many studies, the border between endophytic and mycorrhizal fungi could be difficult to define, and some fungus-plant interactions can easily shift from mutualism to parasitism depending on the plant's physiology and environmental conditions $[45,46,47]$. For the current study, it is worth exploring the effect of $F$. oxysporum TPYD- 6 alone as well as the possible synergistic effect of TPYD-6 with other OMFs on the growth of $D$. officinale seedlings.

\section{Conclusions}

In this study, in situ seedling baiting was used to capture orchid seedling growth-promoting fungi for the first time. Five Tulasnella species and Fusarium oxysporum TPYD-6 were successfully isolated from seedlings of $D$. officinale that were produced in vitro and transplanted for more than one year into their original habitats. Tulasnella sp. TPYD-2 showed a great ability to promote plant growth in $D$. officinale and could be selected for practical use in restoration plantings of $D$. officinale. For any given orchid species, it is still uncertain whether the fungi involved in the seedling stage remain until plant adulthood. Our results suggest that using an in situ/ex situ seedling baiting technique could be an efficient approach to obtain plant growth-promoting fungi. This method might be more reliable for overcollected orchids for which mature plants are hard to find in the wild but their original habitats are known. A similar idea has been used in studies on the interactions between orchids and fungi [48] and suggests that this approach could have broad applications in orchid mycorrhiza studies and orchid conservation. 


\section{Materials And Methods}

\section{Study species and study site}

Dendrobium officinale Kimura \& Migo (synonym of D. catenatum and D. candidum) is a lithophytic orchid widely distributed in subtropical areas, especially karst regions at altitudes from 500 to $1600 \mathrm{~m}$ in China [1]. D. officinale is one of the most popular TCM herbs and is known as Tie-Pi-Shi-Hu in Chinese. The wild populations of $D$. officinale are very small and sparsely distributed due to severe overharvesting over the past 30 years [3], and this species is listed as critically endangered on the IUCN Red List [49]. The study site was located at a limestone mountain near Lengdong village ( $24^{\circ} 54^{\prime} 13^{\prime \prime} \mathrm{N} ; 104^{\circ} 58^{\prime} 43^{\prime \prime} \mathrm{E}$; alt. $1100 \mathrm{~m}$; Fig. 1A) in Xingyi, Guizhou Province, China. This region is a typical karst landform, and more than 20 species of Dendrobium including $D$. officinale have been reported in the area but are seriously threatened due to habitat loss and overcollection [50]. According to local residents, wild plants of $D$. officinale could be found in the mountains of the study site several years ago.

\section{In situ seedling baiting and sampling}

An in situ seedling baiting experiment was conducted using in vitro-produced seedlings of $D$. officinale, in which mature seeds were obtained via hand-outcross pollination of flowers between different individuals of $D$. officinale and asymbiotically geminated on MS medium. The seedlings were removed from the flasks, and the roots were cleaned and then transplanted to the cracks and crevices of calcareous rocks at the study site during May and June at the start of the rainy season in 2016 (Fig. 1B). After more than one year of natural growth, root samples were taken six times at the study site during August 2017 and May 2018 (Additional file 1). For each sampling time, 10 clusters of seedlings containing $3-5$ individuals with robust growth and newly established roots were randomly selected. Ten roots from each cluster were randomly sampled. Collected root samples were placed in an icebox and transferred to the laboratory for fungal isolation on the same day.

\section{Fungal isolation and identification}

The sampled roots were washed with running water, surface sterilized with $75 \%$ ethanol for 2 min and $2 \%$ sodium hypochlorite for $3 \mathrm{~min}$, and then washed three times with distilled water. Mycorrhizal fungi were isolated from roots of $D$. officinale following the method described by Bayman et al. [51]. The superficial uncolonized root tissue was removed using a thin sterile blade, and root fragments containing intracellular pelotons were obtained by scrapping the roots using needles and forceps. The root fragments were scraped as small as possible with an anatomical needle under a 10 * 20 magnification microscope (XS-A, Shanghai Pudan Co., Ltd.), transferred to PDA medium (200 g/L potato, $20 \mathrm{~g} / \mathrm{L}$ dextrose and $20 \mathrm{~g} / \mathrm{L}$ agar) with $0.05 \mathrm{~g} / \mathrm{L}$ penicillin and $0.05 \mathrm{~g} / \mathrm{L}$ streptomycin and incubated at $25 \pm 2{ }^{\circ} \mathrm{C}$. The root fragments were monitored daily during incubation on PDA. When hyphal growth from the root fragments exceeded $0.5 \mathrm{~cm}$ in length, tips of the hyphae were cut and transferred to new PDA medium for purification. After repeating this purification step 4-5 times, purified strains were obtained. 
All obtained fungal strains were morphologically identified and then grouped based on culture characteristics such as colony morphology, hyphal characteristics, hyphal colour, and the colours associated with secondary metabolites [52]. For each group, a strain obtained from each sampling time was randomly selected for molecular identification. The rDNA region containing two ITS regions and the 5.8S gene was amplified using the ITS1 and ITS4 primers [53], and PCR was performed following Selosse et al. [54]. All ITS-rDNA sequences obtained were compared with those deposited in the GenBank database (National Center for Biotechnology Information, NCBI 2012) using the Basic Local Alignment Search Tool (BLAST), which allows the identification of isolates at the genus or species level when the ITS sequence similarity exceeds $95 \%$ or $97 \%$, respectively [55]. Later, one sequence per species was deposited in NCBI GenBank under the accession numbers listed in Table 1.

\section{Phylogenetic analysis}

A phylogenetic tree was generated to visualize the phylogenetic positions of the OMFs obtained in the current study among other known fungal species associated with Dendrobium officinale. By searching scientific databases (e.g., Web of Science; Google Scholar; Weipu Chinese journals database) with the key words "Dendrobium officinale", "Dendrobium catenatum", "Dendrobium candidum" and "mycorrhizal fungi", we collected all the available information on fungi that are considered to have positive effects on D. officinale seedling growth, including original resources, host orchid species and their effects on seedling growth in $D$. officinale (Additional file 2). Then, the available ITS-rDNA sequences of all related representative fungi were downloaded from the GenBank database (https://www.ncbi.nlm.nih.gov/) and used to generate a phylogenetic tree with the fungal sequences obtained in the current study. The ITS sequence of Armillaria sinapina As93 (FJ495039) was set as an outgroup based on previous studies [24]. Alignment of nucleotide sequences was performed by Clustal X version 1.81 [56] followed by manual adjustments using BioEdit [57] (Hall 1999). Maximum likelihood (ML) phylogenetic tree searches and ML bootstrapping were conducted using the web server RAxML-HPC2 on TG ver. 7.2.8 [58, 59] with 1000 rapid bootstrap analyses followed by a search for the best-scoring tree in a single run [58]. Then, FigTree V1.4.3 was used to analyse the resulting RAxML-Bipartitions file. Bayesian inference (BI) was conducted using MrBayes 3.1.2 on Cipres [59, 60, 61], and the MAX time was set from 3 to 4 hours. The resulting file (infile.nex.con.tre) was downloaded, and FigTree v1.4.3 was used for analysis. Then, Figtree v1.4.3 was used to organize the basic form of the phylogenetic tree and export it as a PDF file. Finally, Adobe Illustrator CC 2018 was used for the final modification.

\section{Testing the capacity of fungi to support seedling growth}

The three OMFs with the highest isolation rates were selected, and their capacity to support seedling growth in $D$. officinale was tested. In vitro-produced seedlings of $D$. officinale were transplanted from flasks to circular plastic pots (height * diameter $=8 \mathrm{~cm} * 10 \mathrm{~cm}$ ) after the medium on roots was washed away (Fig. 1C). For each pot, 5 seedlings were planted together in cultivation substrate with the same proportion of volcanic stone, peat and bark fragments. Once seedling transfer was completed, each pot was inoculated with one cubic centimetre of the three fungal inoculants, which were placed in the centres of the pots, to establish three fungal incubation treatments, and a sterile control without fungal 
inoculation was used as a control treatment (CK). Each treatment was replicated in 24 pots, and to avoid possible fungal infection among treatments, all pots from a given treatment were placed in separate germination chambers at $25 \pm 2{ }^{\circ} \mathrm{C}$ with a $12 / 12$-h light/dark cycle.

To determine if and when mycorrhizal symbiosis actually became established, three roots from each treatment were randomly selected and examined for the presence of pelotons at 30,60,90,120, 150 and $180 \mathrm{~d}$ after incubation. Pelotons were cleared using $10 \% \mathrm{KOH}$ solution, washed with $1 \% \mathrm{HCl}$ solution, stained with $0.05 \%(\mathrm{w} / \mathrm{v})$ trypan blue in lactic acid glycerol solution overnight [11], and then destained in acetic glycerol solution before observation under a microscope (DM2000, Leica Microsystems GmbH, Wetzlar, Germany).

\section{Data collection and statistical analysis}

At the same time as seedling transplanting, 40 seedlings were randomly selected to measure the lengths of the longest root and leaf, plant height, and fresh and dry weights. Later, 40 seedlings from each treatment were randomly selected, and the same measurements were made at 120, 150 and $180 \mathrm{~d}$ after incubation. Moreover, to assess the effects of different fungi on seedling growth, the growth parameters including the longest root length $(R)$, longest leaf length $(S)$, plant height $(H)$, fresh weight $(F)$ and dry weight $(D)$ under different fungal treatments at different times were also calculated according to the following formula: $R=\left[\left(\mathrm{R}_{\mathrm{ex}}-\mathrm{R}_{\mathrm{CK}}\right) / \mathrm{R}_{\mathrm{CK}}\right] 100 \%, S=\left[\left(\mathrm{S}_{\mathrm{ex}}-\mathrm{S}_{\mathrm{CK}}\right) / \mathrm{S}_{\mathrm{CK}}\right] 100 \%, H=\left[\left(\mathrm{H}_{\mathrm{ex}}-\mathrm{H}_{\mathrm{CK}}\right) / \mathrm{H}_{\mathrm{CK}}\right] 100 \%, F=\left[\left(\mathrm{F}_{\mathrm{ex}}{ }^{-}\right.\right.$ $\left.\left.\mathrm{F}_{\mathrm{CK}}\right) / \mathrm{F}_{\mathrm{CK}}\right] 100 \%$ and $D=\left[\left(\mathrm{D}_{\mathrm{ex}}-\mathrm{D}_{\mathrm{CK}}\right) / \mathrm{D}_{\mathrm{CK}}\right] 100 \%$. The subscript ex represents different numerical values measured in the different fungal treatments at different times, while the subscript $\mathrm{CK}$ represents the numerical value of the control treatment.

The effects of different OMFs on seedling growth at different time points were studied. The lengths of the longest root and leaf as well as plant height, and fresh and dry weights among the fungal inoculation treatments and control at 120,150 and $180 \mathrm{~d}$ were recorded. Comparisons were made with one-way ANOVA and the least significant difference $(L S D)$ method when the data showed a normal distribution or the generalized linear model (GLM) when the data were not normally distributed. All statistical analyses were performed using SPSS software (version 25.0).

\section{Declarations}

\section{Authors' contributions}

DYC: performed experiments and data analysis; XJW: data collection and phylogenetic analysis; TQL: data analysis; NQL: samples collection and performed experiments; JYG: project design, data analysis and manuscript writing. All authors have read and approved the manuscript.

\section{Funding}


This work was supported by the National Natural Science Foundation of China (Grant No. U1702235) and the Ministry and Province Joint Construction Project of Yunnan University (Grant No. C176280109).

\section{Availability of data and materials}

All data analyzed during this study are included in this published article and its supplementary information files. Additional raw data from sequencing and quality control datasets are available from the corresponding author upon reasonable request.

\section{Ethics approval and consent to participate}

Not applicable.

\section{Consent for publication}

Not applicable.

\section{Competing interests}

The authors declare that they have no competing interests.

\section{References}

1. Chen XQ, Liu ZJ, Zhu GH, Lang KY, Ji ZH, Luo YB, et al. Orchidaceae. In: Wu ZY, Raven PH, Hong DY, editors. Flora of China vol.25. Beijing and St.. Louis: Science press and Missouri Botanical Garden Press; 2009. pp. 367-97.

2. Bao XS, Shun QS, Chen LZ. The medicinal plants of Dendrobium (Shi-Hu) in China. Shanghai: Fudan University Publisher and Shanghai Medical University Publishing House; 2001.

3. Liu H, Luo YB, Heinen J, Bhat M, Liu ZJ. Eat your orchid and have it too: a potentially new conservation formula for Chinese epiphytic medicinal orchids. Biodivers Conserv. 2014;23:1215-28.

4. Liu Q, Chen J, Corlett RT, Fan XL, Yu DL, Yang HP, et al. Orchid conservation in the biodiversity hotspot of southwestern China. Conserv Biol. 2015;29:1563-72.

5. Wilkie DS, Godoy RA. Income and price elasticities of bushmeat demand in lowland Amerindian societies. Conserv Biol. 2001;15:761-9.

6. Cheng J, Dang PP, Zhao Z, Yuan LC, Zhou ZH, Wolfe D, et al. An assessment of the Chinese medicinal Dendrobium industry: Supply, demand and sustainability. J Ethnopharmacol. 2019;229:81-8.

7. Song YQ, Liu ZL, Willian S, Gao JY. Characteristics of the orchid trade at public markets and implications for conservation in Xishuangbanna, Yunnan, China. Biodivers Sci. 2017;25:531-9. 
8. Gao JY, Liu Q, Yu DL. Orchids of Xishuangbanna: diversity and conservation. Beijing: China Forestry Publishing House; 2014.

9. Zi XM, Sheng CL, Goodale UM, Shao SC, Gao JY. In situ seed baiting to isolate germinationenhancing fungi for an epiphytic orchid, Dendrobium aphyllum (orchidaceae). Mycorrhiza. 2014;24:487-99.

10. Huang H, Zi XM, Lin H, Gao JY. Host-specificity of symbiotic mycorrhizal fungi for enhancing seed germination, protocorm formation and seedling development of over-collected medicinal orchid, Dendrobium devonianum. J Microbiol. 2018;56:42-8.

11. Meng YY, Shao SC, Liu SJ, Gao JY. Do the fungi associated with roots of adult plants support seed germination? a case study on Dendrobium exile (Orchidaceae). Glob Ecol Conserv. 2019;17:e00582.

12. Meng YY, Fan XL, Zhou LR, Shao SC, Liu Q, Selosse MA, Gao JY. Symbiotic fungi undergo a taxonomic and functional bottleneck during orchid seeds germination: a case study on Dendrobium moniliforme. Symbiosis. 2019;79:205-12.

13. Shao SC, Burgess KS, Cruse-Sanders JM, Liu Q, Fan XL, Huang H, et al. Using in situ symbiotic seed germination to restore over-collected medicinal orchids in southwest China. Front Plant Sci. 2017;8:888.

14. McCormick MK, Whigham DF, O'Neill J. Mycorrhizal diversity in photosynthetic terrestrial orchids. New Phytol. 2004;163:425-38.

15. Rafter M, Yokoya K, Schofield EJ, Zettler LW, Sarasan V. Non-specific symbiotic germination of Cynorkis purpurea (Thouars) Kraezl., a habitat-specific terrestrial orchid from the central highlands of Madagascar. Mycorrhiza. 2016;26:541-52.

16. Těšitelová T, Těšitel J, Jersáková J, R`íhová G, Selosse MA. Symbiotic germination capability of four Epipactis species (Orchidaceae) is broader than expected from adult ecology. Am J Bot. 2012;99:1020-32.

17. Smith SE, Read D. Mycorrhizal symbiosis. London: Academic Press; 2008.

18. Rasmussen HN, Rasmussen FN. Orchid mycorrhiza: implications of a mycophagous life style. Oikos. 2009;118:334-5.

19. Dearnaley JDW, Martos F, Selosse MA. Orchid mycorrhizas: molecular ecology, physiology, evolution and conservation aspects. In: Hock B, editor. The Mycota. vol. 9. Berlin: Springer; 2012. pp. 207-30.

20. Bonfante $P$, Genre A. Mechanisms underlying beneficial plant-fungus interactions in mycorrhizal symbiosis. Nat Commun. 2010;1:48.

21. van der Heijden MGA, Martin FM, Selosse MA, Sanders IR. Mycorrhizal ecology and evolution: the past, the present, and the future. New Phytol. 2015;205:1406-23.

22. Khare E, Mishra J, Arora NK. Multifaceted interactions between endophytes and plant: developments and prospects. Front Microbiol. 2018;9:2732.

23. Favre-Godal Q, Gourguillon L, Lordel-Madeleine S, Gindro K, Choisy P. Orchids and their mycorrhizal fungi: an insufficiently explored relationship. Mycorrhiza. 2020;30:5-22. 
24. Tan XM, Wang CL, Chen XM, Zhou YQ, Wang YQ, Luo AX, et al. In vitro seed germination and seedling growth of an endangered epiphytic orchid, Dendrobium officinale, endemic to China using mycorrhizal fungi (Tulasnella sp.). Sci Hortic. 2014;165:62-8.

25. Teixeira da Silva JA, Tsavkelova EA, Zeng SJ, Ng TB, Parthibhan S, Dobranszki J, et al. Symbiotic in vitro seed propagation of Dendrobium: fungal and bacterial partners and their influence on plant growth and development. Planta. 2015;242:1-22.

26. Zettler LW, Hofer CJ. Propagation of the little club-spur orchid (Platanthera clavellata) by symbiotic seed germination and its ecological implications. Environ Exp Bot. 1998;39:189-95.

27. Sebastián F, Vanesa S, Eduardo F, Graciela T, Silvana S. Symbiotic seed germination and protocorm development of Aa achalensis Schltr., a terrestrial orchid endemic from Argentina. Mycorrhiza. 2014;24:35-43.

28. Shao SC, Xi HP, Mohandass D. Symbiotic mycorrhizal fungi isolated via ex situ seed baiting induce seed germination of Dendrobium catenatum Lindl. (Orchidaceae). Appl Ecol Env Res. 2019;17:975371.

29. Bidartondo MI, Read DJ. Fungal specificity bottlenecks during orchid germination and development. Mol Ecol. 2008;17:3707-16.

30. Long JRD, Swarts ND, Dixon KW, Egerton-Warburton LM. Mycorrhizal preference promotes habitat invasion by a native Australian orchid: Microtis media. Ann Bot. 2013;111:409-18.

31. Rasmussen HN, Whigham DF. Seed ecology of dust seeds in situ: a new study technique and its application to terrestrial orchids. Am J Bot. 1993;80:1374-8.

32. Brundrett MC, Scade A, Batty AL, Dixon KW, Sivasithamparam K. Development of in situ and ex situ seed baiting techniques to detect mycorrhizal fungi from terrestrial orchid habitats. Mycol Res. 2003;107:1210-20.

33. Meng YY, Zhang WL, Selosse MA, Gao JY. Are fungi from adult orchid roots the best symbionts at germination? A case study. Mycorrhiza. 2019;29:541-7.

34. Yang WK, Li TQ, Wu SM, Finnegan PM, Gao JY. Ex situ seed baiting to isolate germination-enhancing fungi for assisted colonization in Paphiopedilum spicerianum, a critically endangered orchid in China. Glob Ecol Conserv. 2020;23:e01147.

35. Zhang Y, Li YY, Chen XM, Guo SX, Lee YI. Effect of different mycobionts on symbiotic germination and seedling growth of Dendrobium officinale, an important medicinal orchid. Bot Stud. 2020;61:2.

36. Bayman P, Otero JT. Microbial endophytes of orchid roots. In: Schulz BJE, Boyle CJC, Sieber TN, editors. Microbial root endophytes. Berlin Heidelberg: Springer; 2006. pp. 153-77.

37. Yuan ZL, Chen YC, Yang Y. Diverse non-mycorrhizal fungal endophytes inhabiting an epiphytic, medicinal orchid (Dendrobium nobile): estimation and characterization. World J Microbiol Biotechnol. 2009;125:295-303.

38. Chutima R, Dell B, Vessabutr S, Bussaban B, Lumyong S. Endophytic fungi from Pecteilis susannae (L.) Rafifin (Orchidaceae), a threatened terrestrial orchid in Thailand. Mycorrhiza. 2011;21:221-9. 
39. Srivastava S, Kadooka C, Uchida JY. Fusarium species as pathogen on orchids. Microbiol Res. 2018;207:188-95.

40. Zhang YQ, Lin BY, Zou MY, Liang JX, Hu HQ. First report of Fusarium wilt of Dendrobium officinale caused by Fusarium oxysporum in China. Plant Dis. 2017;101:1039.

41. Vujanovic V, St-Arnaud M, Barabe D, Thibeault G. Viability testing of orchid seed and the promotion of colouration and germination. Ann Bot. 2000;86:79-86.

42. Chen XM, Dong HL, Hu KX, Sun ZR, Chen J, Guo SX. Diversity and antimicrobial and plant-growthpromoting activities of endophytic fungi in Dendrobium loddigesii Rolfe. J Plant Growth Regul. 2010;29:328-37.

43. Xing YM, Chen J, Cui JL, Chen XM, Guo SX. Antimicrobial activity and biodiversity of endophytic fungi in Dendrobium devonianum and Dendrobium thyrsiflorum from Vietman. Curr Microbiol. 2011;62:1218-24.

44. Jiang JW, Zhang K, Cheng S, Nie QW, Zhou SX, Chen QQ, et al. Fusarium oxysporum KB-3 from Bletilla striata: an orchid mycorrhizal fungus. Mycorrhiza. 2019;29:531-40.

45. Johnson NC, Graham JH, Smith FA. Functioning of mycorrhizal associations along the mutualism parasitism continuum. New Phytol. 1997;135:575-85.

46. Bender SF, da Valadares RBS, Taudiere A. Mycorrhizas: dynamic and complex networks of power and influence. New Phytol. 2014;204:15-8.

47. Selosse MA, Schneider-Maunoury L, Martos F. Time to re-think fungal ecology? Fungal ecological niches are often prejudged. New Phytol. 2018;217:968-72.

48. Cevallos S, Declerck S, Suárez JP. In situ orchid seedling-trap experiment shows few keystone and many randomly associated mycorrhizal fungal species during early plant colonization. Front Plant Sci. 2018;9:1664.

49. China Plant Specialist Group. Dendrobium officinale. In: The IUCN Red List of Threatened Species. 2004. doi:10.2305/IUCN.UK.2004.RLTS.T46601A11068443.en.

50. Luo XQ, Wu MK, Zhang XB, Cha LS, Ao MH. Resources status and continuous drought impact assessment on Dendrobium in Southwest Guizhou. J South Agr. 2013;44:1424-30.

51. Bayman P, Espinosa ATM, Aponte CMS, Guevara NCH, Ruiz NLV. Age-dependent mycorrhizal specificity in an invasive orchid, Oeceoclades maculata. Am J Bot. 2016;103:1880-9.

52. Prakash PY, Bhargava K. A modified micro chamber agar spot slide culture technique for microscopic examination of filamentous fungi. J Microbiol Methods. 2016;123:126-9.

53. White TJ, Bruns T, Lee S, Taylor JW. Amplification and direct sequencing of fungal ribosomal RNA genes for phylogenetics. In: Innis MA, Gelfand DH, Sninsky JJ, White TJ, editors. PCR protocols: a guide to methods and applications. New York: Academic; 1990. pp. 315-22.

54. Selosse MA, Weiss M, Jany JL, Tillier A. Communities and populations of sebacinoid basidiomycetes associated with the achlorophyllous orchid Neottia nidusavis (L.) L.C.M. Rich. and neighbouring tree ectomycorrhizae. Mol Ecol. 2002;11:1831-44. 
55. Sánchez Márquez S, Bills GF, Zabalgogeazcoa I. Diversity and structure of the fungal endophytic assemblages from two sympatric coastal grasses. Fungal Divers. 2008;33:87-100.

56. Thompson JD, Gibson TJ, Plewniak F, Jeanmougin F, Higgins DG. The CLUSTAL_X windows interface: flexible strategies for multiple sequence alignment aided by quality analysis tools. Nucleic Acids Res. 1997;25:4876-826.

57. Hall TA. BioEdit: a user-friendly biological sequence alignment editor. and analysis program for Windows 95/98/NT. In: Nucleic acids symposium series vol. 41. London: Information Retrieval Ltd.; 1999. p. 95-98.

58. Stamatakis A, Hoover P, Rougemont J. A rapid bootstrap algorithm for the RAxML web servers. Syst Biol. 2008;57:758-71.

59. Miller MA, Pfeiffer W, Schwartz T. Creating the CIPRES Science Gateway for inference of large phylogenetic trees. In: 2010 gateway computing environments workshop; 2010. p. 1-8.

60 . Huelsenbeck JP, Ronquist F. MRBAYES: Bayesian inference of phylogenetic trees. Bioinformatics. 2001;17:754-5.

61. Ronquist F, Huelsenbeck JP. MrBayes 3: Bayesian phylogenetic inference under mixed models. Bioinformatics. 2003;19:1572-4.

62. Fujimori S, Abe JP, Okane I, Yamaoka Y. Three new species in the genus Tulasnella isolated from orchid mycorrhiza of Spiranthes sinensis var. amoena (Orchidaceae). Mycoscience. 2018;60: 71-81.

63. Yuan L, Yang ZL, Li SY, Hu H, Huang JL. Mycorrhizal specificity, preference, and plasticity of six slipper orchids from South Western China. Mycorrhiza. 2010;20:559-68.

64. Katoch M, Phull S, Vaid S, Singh S. Diversity, phylogeny, anticancer and antimicrobial potential of fungal endophytes associated with Monarda citriodora L. BMC Microbiol. 2017;17:44-57.

\section{Figures}



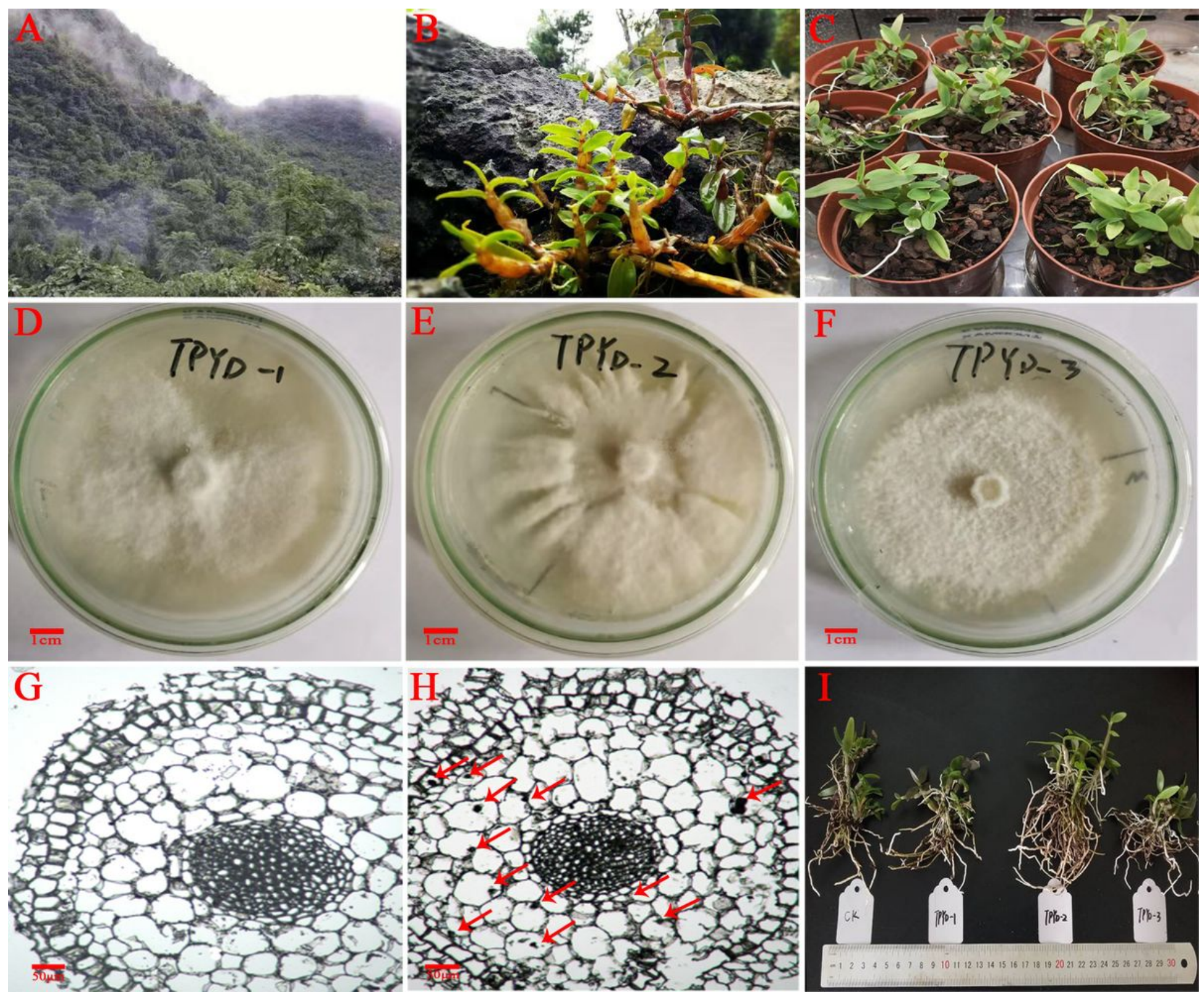

Figure 1

A, The study site, which is a limestone mountain and part of the original habitat of Dendrobium officinale. $B$, In vitro-produced seedlings of D. officinale transplanted on rocks at the study site. C, Potted in vitroproduced seedlings of $\mathrm{D}$. officinale used to test the abilities to fungi to promote seedling growth by inoculation with different fungal strains. D, A colony of fungal strain TPYD-1. E, A colony of fungal strain TPYD-2. F, A colony of fungal strain TPYD-3. G, Cross sections of D. officinale seedling roots at $60 \mathrm{~d}$ after incubation showing no pelotons established in the roots under any treatment. $\mathrm{H}$, Cross section of seedling roots of $D$. officinale at $180 \mathrm{~d}$ after incubation showing pelotons (red arrows) established in the roots under the three fungal treatments. I, Seedlings under different fungal treatments and the control treatment at $180 \mathrm{~d}$ after incubation showing that the fungus TPYD-2 can effectively promote growth of $D$. officinale seedlings. 


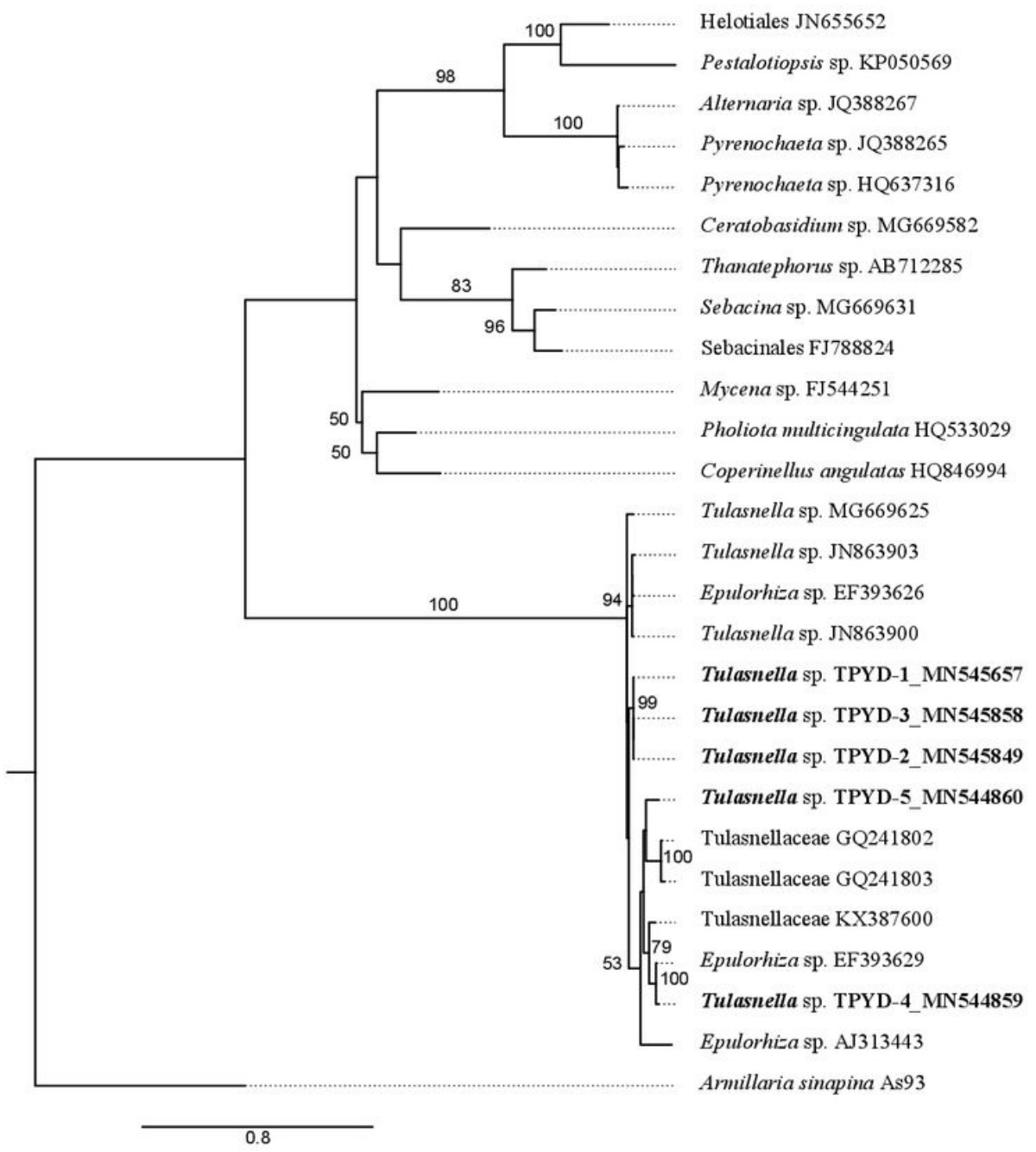

\section{Figure 2}

Maximum-likelihood tree constructed using ITS sequences of five Tulasnella species (in bold) obtained from in situ baiting of Dendrobium officinale seedlings in this study and of other fungi reported to have positive effects on the growth of $D$. officinale seedlings. Numbers above branches are bootstrap probabilities (whenever $\geq 50 \%$ ) out of 1000 replicates. 

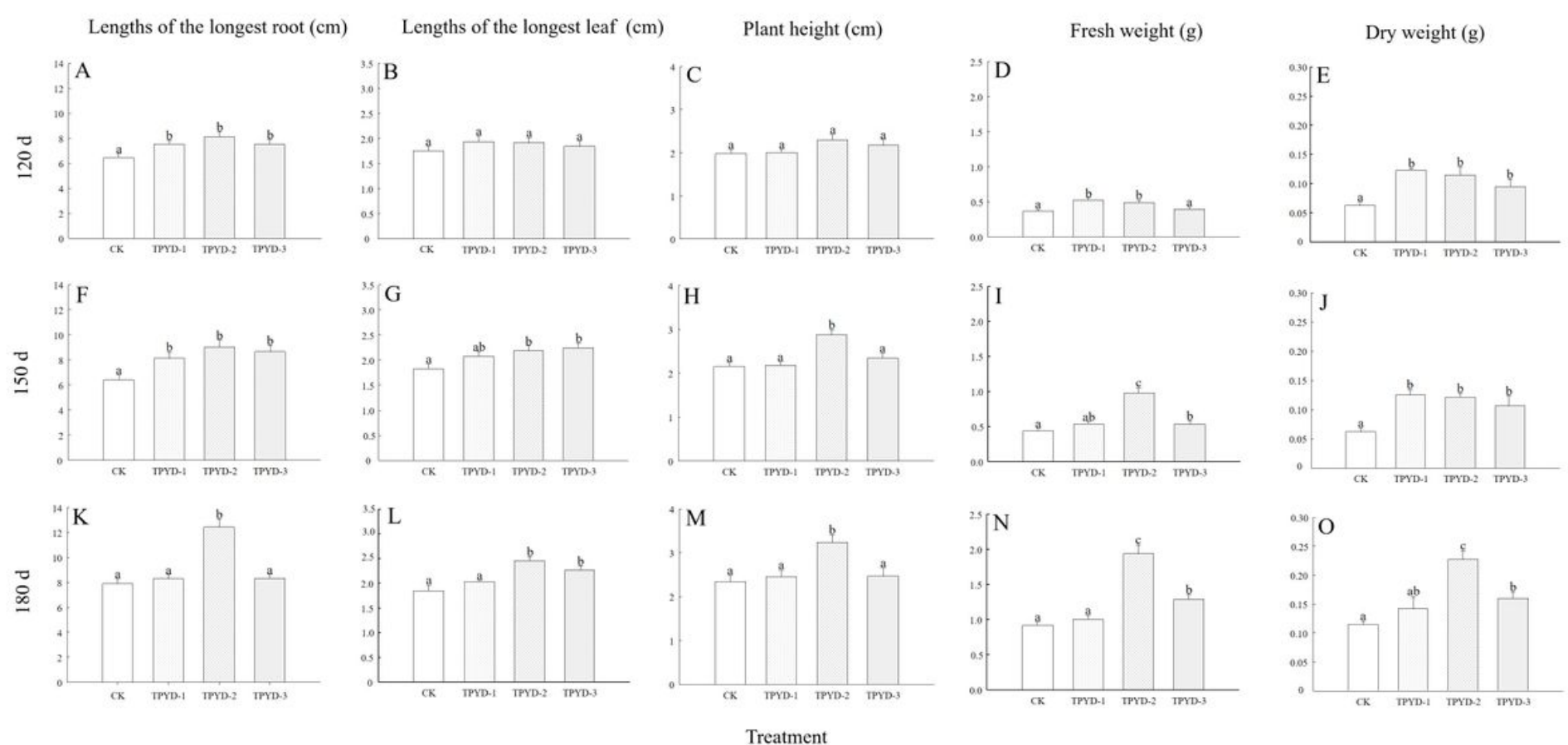

\section{Figure 3}

Effects on the length of the longest root, length of the longest leaf, plant height, fresh weight and dry weight from different treatments (control on MS medium or OMA with addition of one of the three Tulasnella isolates - TPYD-1, TPYD-2 or TPYD-3) at 120, 150 and $180 \mathrm{~d}$ after incubation. In each panel, different letters indicate significant differences based on one-way ANOVA and the least significant difference (LSD) method where the data show a normal distribution and the generalized linear model (GLM) where the data are not normally distributed.

\section{Supplementary Files}

This is a list of supplementary files associated with this preprint. Click to download.

- Additionalfile1.docx

- Additionalfile2.docx 